\title{
Global Age Distribution of Pediatric Norovirus Cases
}

Running head: Age distribution of norovirus cases

Authors: Kayoko Shioda, ${ }^{\mathrm{a}, \mathrm{b}}$, Anita Kambhampati ${ }^{\mathrm{a}, \mathrm{b}}$, Aron J. Hall ${ }^{\mathrm{a}}$, Ben A. Lopman ${ }^{\mathrm{a}}$

\author{
Author affiliations: \\ ${ }^{a}$ National Center for Immunization and Respiratory Diseases, Centers for Disease Control and \\ Prevention, Atlanta, Georgia 30329 USA \\ ${ }^{\mathrm{b}}$ Oak Ridge Institute for Science and Technology, Oak Ridge, TN 37831 USA
}

\author{
Authors' email addresses: \\ Kayoko Shioda: yji4@cdc.gov \\ Anita Kambhampati: wyc4@cdc.gov \\ Aron Hall: esg3@cdc.gov \\ Ben Lopman: $\underline{\text { iow4 @ cdc.gov }}$
}

Corresponding author: Kayoko Shioda, DVM, MPH, National Center for Immunization and

Respiratory Diseases, Centers for Disease Control and Prevention, 1600 Clifton Road NE,

Atlanta, GA 30333 USA, Email: yji4@ @dc.gov, Telephone: (404) 639-0002

Word count: 138 words (abstract), 1501 words (text) 


\section{INTRODUCTION}

With the substantial decline of rotavirus-associated diarrhea in countries that have introduced rotavirus vaccines, norovirus is increasingly recognized as a main cause of acute gastroenteritis (AGE) $[1,2]$. Norovirus vaccines are under development and have shown promise in safety and immunogenicity studies, as well as protection against infection and disease in experimental challenge studies [3,4]. Young children have the highest incidence of norovirus gastroenteritis $[5,6]$, so stand to benefit from a vaccine. However all vaccine studies have been performed among adults. For development of a pediatric vaccine, a number of specific questions will arise, including the number of doses required, the acceptability of an adjuvant, and the appropriate age to vaccinate. To identify the most critical age periods to prevent norovirus among young children, we conducted meta-analysis to understand the detailed age distribution of pediatric norovirus AGE cases (defined as children aged $<5$ years).

\section{METHODS}

We used the database described in a previous systematic review [7] that included studies published between January 1997 and March 2014; we updated the literature search to include studies published between March 1, 2014, and August 31, 2014 in the Medline database. We searched for studies using the search term "norovirus." Two individuals reviewed titles and abstracts and obtained full articles if studies were deemed relevant to our research. Studies that met all four of the following criteria were included: (1) recruited patients with AGE symptoms from a specific geographic area or group of people; (2) used PCR to detect norovirus in stool; (3) done continuously for >1 year; and (4) reported the age distribution among laboratory-confirmed pediatric norovirus cases. 
Summary of age distributions

Data on the total number of cases and the number of cases in each age group were

27 extracted from each study. After trying various categorizations (Supplementary Table/Figure

28 $1 \& 2)$, the following four age groups were used for analyses: <6, 6-11, 12-23, and 24-59 months.

The cumulative proportion of pediatric norovirus cases by age was calculated for each study. We pooled this cumulative proportion of cases and calculated a weighted average. The following formula shows the weighted average of the cumulative proportion of pediatric norovirus cases by the age of 6 months:

$$
=\frac{\sum_{i}\left(\text { Cases }_{i} * P(\text { Cases } 0-5 m)_{i}\right)}{\sum_{i} \operatorname{Cases}_{i}}
$$

where $P(\text { Cases } 0-5 m)_{i}$ is the proportion of pediatric norovirus cases that were among the $<6$ month age group and Cases $_{i}$ is the total number of norovirus cases aged 0-59 months in study $i$. $\sum_{i}$ Cases $_{i}$ is the sum of all norovirus cases 0-59 months of age for all included studies. Similar formulae were used to calculate weighted average for $<12$ and $<24$ month age groups.

\section{Association of age distribution with socio-demographic factors}

Per capita gross domestic product (GDP) of each country from the year of publication was used as an indicator of income level [8]. Countries were additionally categorized into three levels of development according to World Health Organization (WHO) mortality strata [9]: highmortality developing (HMD), low-mortality developing (LMD), and developed. The study settings were grouped into inpatient (including emergency departments), outpatient, community, and other. The "other" category included studies in which setting was not described or a mixture of settings was included but stratified data were not reported. 
We used meta-regression analyses to evaluate associations between potential predictors

47 (i.e., income level and development index of each country and study setting) with the proportion

48 of pediatric cases that occurred in the $<12$ month age group. We selected this age limit as the

49 outcome because the largest variation in cumulative proportion of cases across socio-

50 demographic factors was observed at 12 months. Mixed-effects models were fitted using

51 restricted maximum-likelihood estimation, with the number of pediatric norovirus AGE cases in

52 each study as weights and "study" as a random effect. We assumed that per capita GDP and

53 WHO mortality strata would indicate similar levels of development; thus we fit a multivariate

54 mixed-effects model with per capita GDP and study setting. All analyses were performed using

55 the package "metafor" in R [10].

56

\section{RESULTS}

We identified 78 studies about pediatric norovirus from the previously developed database [7]. We also identified 261 publications published from March to August 2014 from the

60

61

62

63

64

65

66

67
Medline database search. Of these, 225 articles were excluded after screening titles and abstracts.

We assessed the eligibility of 114 full-text articles in total. Of these, 79 were excluded for various reasons (Supplementary Figure 3). In total, 35 articles met the inclusion criteria.

There were 23 countries represented in these 35 papers. Twenty (57\%) studies were conducted in LMD countries, representing a total of 2,420 pediatric norovirus cases, eight (23\%) were in developed countries, representing 2,173 cases, and seven were in HMD countries, representing 607 cases. In total, there were 2,347 pediatric norovirus cases in the inpatient setting, 1,329 in outpatient, 530 in community, and 994 in other settings. 
After weighting by the number of pediatric norovirus cases in each study, the percentage

87 of cases in each age group was as follows: 0-5 months: $13 \%$ (95\% confidence interval (CI): 719\%); 6-11 months: 33\% (46\% cumulative; 95\% CI: 36-57\%); 12-23 months: 36\% (82\% cumulative; 95\% CI: 74-90\%); and 24-59 months: 18\% (100\% cumulative) (Figure A\&B). A younger age distribution of pediatric norovirus cases was observed in studies pooled from developing countries, with 60\% (95\% CI: 41-79\%), 52\% (95\% CI: 43-61\%), and 35\% (95\% CI: $12-57 \%$ ) of cases among children < 12 months in HMD, LMD, and developed countries, respectively ( $\mathrm{p}=0.09$, Table and Figure $\mathrm{C})$. A younger age distribution was observed in low income countries, with a decrease of $5.9 \%$ (95\% CI: $1.6-10.1 \%)$ in cumulative cases $<12$ months for every 10,000 USD increase in per capita GDP (Table and Supplementary Figure 4). The age distribution was younger in inpatient settings with 50\% (95\% CI: 42-59\%) of cases aged $<12$ months, compared to 37\% (95\% CI: 16-57\%) in outpatient settings and $24 \%$ (95\% CI: $0.6-54 \%)$ in community settings $(\mathrm{p}=0.01$, Table and Figure $\mathrm{D})$.

After controlling for study setting in the multivariate model, per capita GDP remained significant, with the cumulative proportion of cases by 12 months reduced by $4.1 \%$ (95\% CI: $0.8-7.5 \%$ ) for every 10,000 USD increase in per capita GDP (Table). Although not statistically significant, the cumulative proportion of cases aged <12 months in inpatient settings was $14 \%$ higher than that in outpatient settings in developed countries, while there was no difference in developing countries (Supplementary Figure 5).

\section{DISCUSSION}

Approximately $70 \%$ of pediatric norovirus-associated AGE cases occurred within the 623 month age range, with less than $15 \%$ occurring before 6 months. We found a younger age 
91 distribution of pediatric norovirus cases in lower income settings. This might be attributable to a

92 higher force of norovirus infection/basic reproduction number $\left(\mathrm{R}_{0}\right)$ in low income settings due to

93 poorer hygiene and sanitation and/or higher levels of contact between individuals [11]. These

94 findings suggest that a norovirus immunization schedule completed by 6 months could have the 95 potential to prevent about $85 \%$ of pediatric cases, while a vaccine delivered at 12 months of age 96 would only have the potential to prevent about $50 \%$. With a younger age distribution in lower 97 income settings, early prevention is even more critical.

The proportion of cases among children aged $<12$ months increased from community to 99 outpatient to inpatient settings. Assuming setting correlates with severity of illness, this suggests that the age distribution of severe norovirus cases is younger than mild cases and that younger 101 cases are more likely to be hospitalized or seek medical care, consistent with previous studies $102[12,13]$. This trend was apparent (though non-significant) in developed countries but not in 103 developing countries. This could reflect more access to care for mild cases in developed 104 countries, or a clearer triage of cases in inpatient versus outpatient settings. Also, there may be 105 biases in surveillance in the individual studies that affected case ascertainment. For example, if 106 older children are less likely to be hospitalized in developing countries, either because they do 107 not seek care, or because they are triaged as outpatients, then we would observe an older 108 distribution in developed country settings. If true, our observed differences could be a result of 109 care-seeking and case detection rather than disease severity. An important limitation was that few included studies were conducted in HMD countries 111 or community settings, which may limit the representativeness of age distribution of these 112 subgroups in the aggregated results. Also, we could not assess the age distribution by genogroup 113 due to limitations in the published data, and this may be important since routes of GI and GII 
114 differ somewhat, and, therefore, may be associated with different age at first infection.

115 Additionally, we only included studies which used PCR for norovirus detection. While our

116 inclusion criteria also required cases to demonstrate AGE symptoms, the detection of norovirus

117 by PCR does not necessarily imply that AGE in every case was caused by norovirus.

118 In conclusion, we found that a majority of pediatric norovirus AGE cases occurred

119 between 6 and 24 months. Norovirus vaccines are progressing through the development pipeline;

120 our review makes clear that a pediatric formulation would need to be delivered by 6 months of

121 age to prevent the majority of pediatric norovirus cases.

122

that might pose a conflict of interest.

Disclaimer. The findings and conclusions presented in this paper are of the authors and

126 do not necessarily represent the Centers for Disease Control and Prevention.

Acknowledgements. This research was supported by (a) an appointment to the Research

128 Participation Program at the Centers for Disease Control and Prevention administered by the Oak

129 Ridge Institute for Science and Education through an interagency agreement between the U.S.

130 Department of Energy and CDC (to K.S. and A.K.) and (b) the Foodborne Disease Burden

131 Epidemiology Reference Group (FERG) of the World Health Organization (WHO). We thank

132 Marion Koopmans and Linda Verhoef at the National Institute for Public Health and the

133 Environment in the Netherlands for their previous contribution to developing the database. 


\section{REFERENCES}

[1] Payne DC, Vinje J, Szilagyi PG, Edwards KM, Staat MA, Weinberg GA, et al. Norovirus and medically attended gastroenteritis in U.S. children. N Engl J Med 2013 Mar 21;368(12):1121-30.

[2] Hemming M, Rasanen S, Huhti L, Paloniemi M, Salminen M, Vesikari T. Major reduction of rotavirus, but not norovirus, gastroenteritis in children seen in hospital after the introduction of RotaTeq vaccine into the National Immunization Programme in Finland. Eur J Pediatr 2013 Jun;172(6):739-46.

[3] Atmar RL, Bernstein DI, Harro CD, Al-Ibrahim MS, Chen WH, Ferreira J, et al. Norovirus vaccine against experimental human Norwalk Virus illness. N Engl J Med 2011 Dec 8;365(23):2178-87.

[4] El-Kamary SS, Pasetti MF, Mendelman PM, Frey SE, Bernstein DI, Treanor JJ, et al. Adjuvanted intranasal Norwalk virus-like particle vaccine elicits antibodies and antibodysecreting cells that express homing receptors for mucosal and peripheral lymphoid tissues. J Infect Dis 2010 Dec 1;202(11):1649-58.

[5] Phillips G, Tam CC, Conti S, Rodrigues LC, Brown D, Iturriza-Gomara M, et al. Community incidence of norovirus-associated infectious intestinal disease in England: improved estimates using viral load for norovirus diagnosis. Am J Epidemiol 2010 May 1;171(9):1014-22.

[6] Hall AJ, Lopman BA, Payne DC, Patel MM, Gastanaduy PA, Vinje J, et al. Norovirus disease in the United States. Emerg Infect Dis 2013 Aug;19(8):1198-205.

[7] Ahmed SM, Hall AJ, Robinson AE, Verhoef L, Premkumar P, Parashar UD, et al. Global prevalence of norovirus in cases of gastroenteritis: A systematic review and meta-analysis. Lancet Infect Dis 2014 August;14(8):725-30. 
[8] World Bank. GDP per capita (current US\$). 2014 [cited; Available from: http://data.worldbank.org/indicator/NY.GDP.PCAP.CD

[9] World Health Organizaiton. List of Member States by WHO region and mortality stratum. 2007 [cited; Available from:

http://www.who.int/mental_health/neurology/annexes_neuro_disorders_public_h_challenges.pdf

[10] Viechtbauer W. Conducting meta analysis in R with the metafor package. Journal of Statistical Software 2010;36(3):1-48.

[11] Fine PE, Carneiro IA. Transmissibility and persistence of oral polio vaccine viruses: implications for the global poliomyelitis eradication initiative. Am J Epidemiol 1999 Nov 15;150(10):1001-21.

[12] Patel AB, Ovung R, Badhoniya NB, Dibley MJ. Risk factors for predicting diarrheal duration and morbidity in children with acute diarrhea. Indian journal of pediatrics 2012 Apr;79(4):472-7.

[13] Rocha MC, Carminate DL, Tibirica SH, Carvalho IP, Silva ML, Chebli JM. Acute diarrhea in hospitalized children of the municipality of Juiz de Fora, MG, Brazil: prevalence and risk factors associated with disease severity. Arquivos de gastroenterologia 2012 Dec;49(4):25965. 
A
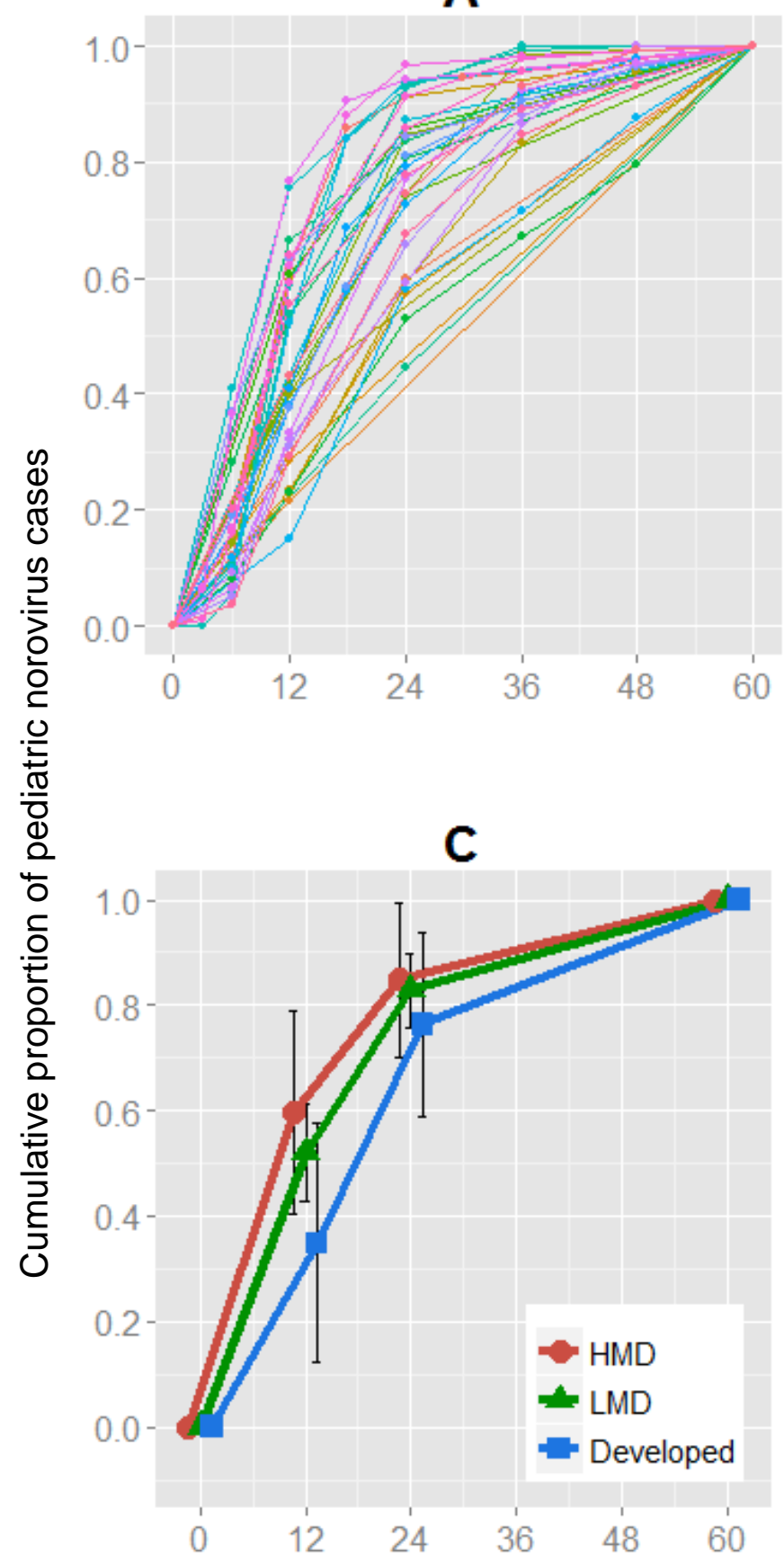

B

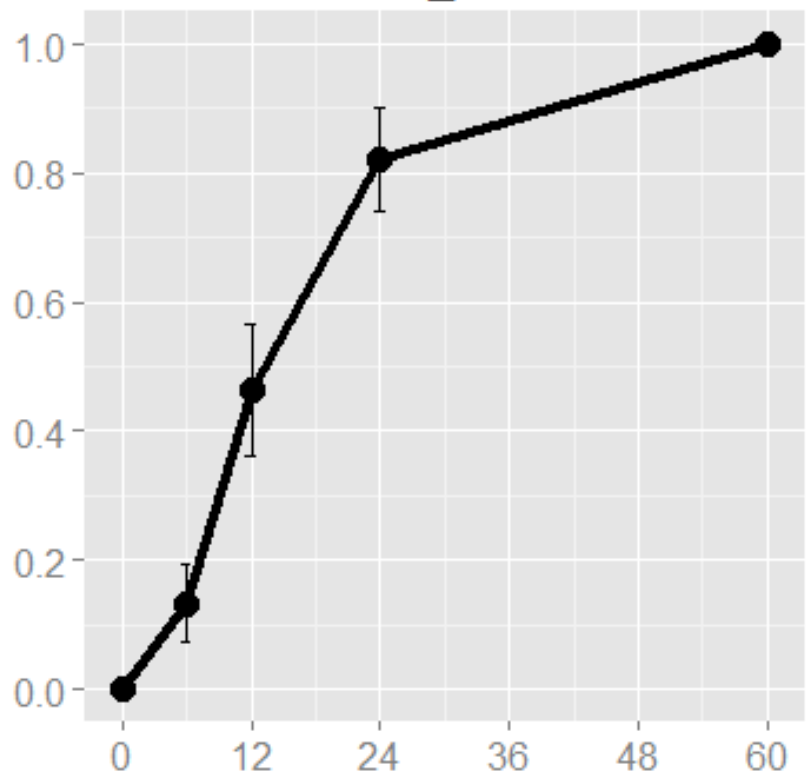

D

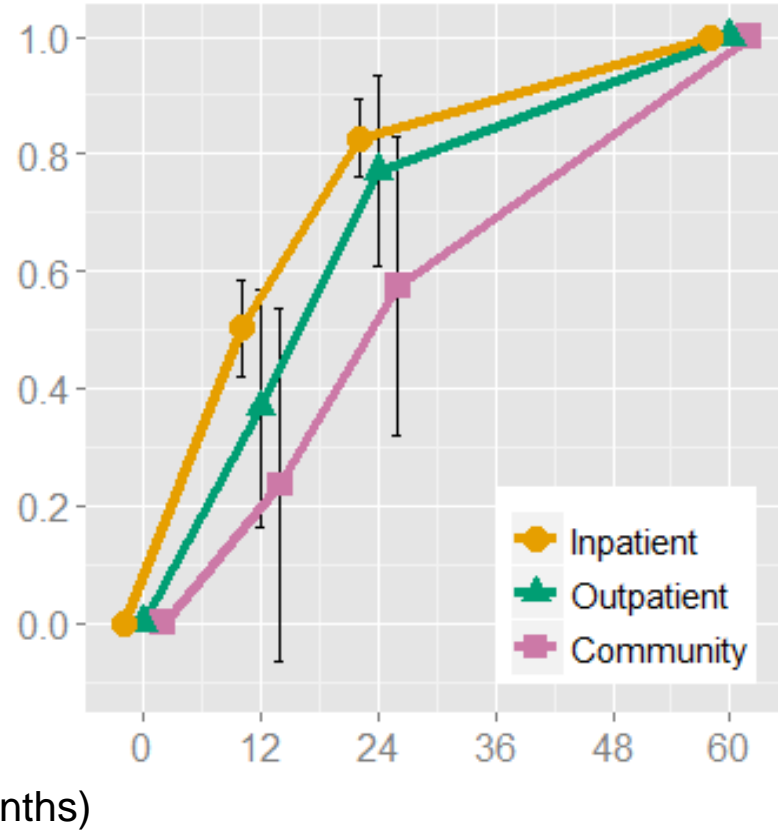


Table: Bivariate and multivariate associations between the predictors and the cumulative proportion of pediatric cases by 12 month of age ${ }^{\mathrm{a}}$

\begin{tabular}{|c|c|c|c|c|c|c|}
\hline \multirow{2}{*}{$\begin{array}{l}\text { Predictors } \\
\text { WHO mortality strata }\end{array}$} & \multicolumn{3}{|c|}{ Bivariate association } & \multicolumn{3}{|c|}{ Multivariate association } \\
\hline & \multicolumn{2}{|c|}{ Parameter estimate (se) } & p-value & \multicolumn{2}{|c|}{ Parameter estimate (se) } & p-value \\
\hline HMD & 0.27 & $(0.13)$ & 0.04 & & - & - \\
\hline LMD & 0.19 & $(0.10)$ & 0.06 & & - & - \\
\hline Developed & Ref & & & & - & - \\
\hline \multicolumn{7}{|l|}{ Settings } \\
\hline Outpatient & -0.14 & $(0.11)$ & 0.21 & -0.05 & $(0.10)$ & 0.6 \\
\hline Community & -0.25 & $(0.11)$ & 0.02 & -0.16 & $(0.10)$ & 0.1 \\
\hline Other $^{b}$ & 0.14 & $(0.09)$ & 0.13 & 0.13 & $(0.09)$ & 0.1 \\
\hline Inpatient & Ref & & & Ref & & \\
\hline Per capita GDP (per 10,000 USD) & -0.06 & $(0.02)$ & 0.007 & -0.04 & $(0.02)$ & 0.02 \\
\hline
\end{tabular}

Abbreviations: se, standard error; HMD, high-mortality developing countries; LMD, low-mortality developing countries; Ref, reference group; GDP, gross domestic product; USD, United States Dollars.

${ }^{\text {a }}$ Note that a negative coefficient denotes a lower proportion of cases less than 12 month of age in a given category compared to the reference group.

b The "other" settings category included studies in which setting was not described or in which a mixture of settings was included but stratified data were not reported. 